\title{
Spin-locking of half-integer quadrupolar nuclei in NMR of solids: The far off-resonance case
}

\author{
Smita Odedra ${ }^{\mathrm{a}}$, Stephen Wimperis ${ }^{\mathrm{b}, *}$ \\ ${ }^{a}$ School of Chemistry, University of Glasgow, Glasgow G12 8QQ, United Kingdom \\ ${ }^{b}$ Department of Chemistry, Lancaster University, Lancaster LA1 4YB, United Kingdom
}

\begin{abstract}
Spin-locking of spin $I=3 / 2$ and $I=5 / 2$ nuclei in the presence of large resonance offsets has been studied using both approximate and exact theoretical approaches and, in the case of $I=3 / 2$, experimentally. We show the variety of coherences and population states produced in a far off-resonance spin-locking NMR experiment (one consisting solely of a spin-locking pulse) and how these vary with the radiofrequency field strength and offset frequency. Under magic angle spinning (MAS) conditions and in the "adiabatic limit", these spin-locked states acquire a time dependence. We discuss the rotor-driven interconversion of the spin-locked states, using an exact density matrix approach to confirm the results of the approximate model. Using conventional and multiple-quantum filtered spin-locking ${ }^{23} \mathrm{Na}(I=3 / 2) \mathrm{NMR}$ experiments under both static and MAS conditions, we confirm the results of the theoretical calculations, demonstrating the applicability of the approximate theoretical model to the far off-resonance case. This simplified model includes only the effects of the initial rapid dephasing of coherences that occurs at the start of the spin-locking period and its success in reproducing both experimental and exact simulation data indicates that it is this dephasing that is the dominant phenomenon in NMR spin-locking of quadrupolar nuclei, as we have previously found for the on-resonance and near-resonance cases. Potentially, far off-resonance spin-locking of quadrupolar
\end{abstract}

\footnotetext{
* Corresponding author

Email address: s.wimperis@lancaster.ac.uk (Stephen Wimperis)
} 
nuclei could be of interest in experiments such as cross polarisation as a consequence of the spin-locking pulse being applied to a better defined initial state (the thermal equilibrium bulk magnetisation aligned along the $z$-axis) than can be created in a powdered solid with a selective radiofrequency pulse, where the effect of the pulse depends on the orientation of the individual crystallites.

Keywords: Quadrupolar nuclei, Spin-locking, Off-resonance

irradiation, Cross polarisation, ${ }^{23} \mathrm{Na}$

\section{Introduction}

The spin-locking experiment in NMR involves the application of a long radiofrequency ( $\mathrm{rf}$ ) pulse in the direction of the bulk magnetisation vector in the rotating frame, with the effect of locking the vector in place for up to several 5 seconds. No further evolution takes place under the influence of resonance offsets, $J$ coupling or dipolar coupling while the magnetisation is spin-locked, as the radiofrequency field strength $\left(\omega_{1}=\left|\gamma B_{1}\right|\right)$ is much stronger than any of these interactions. This spin-locking is an essential part of many experiments in NMR, most notably the cross-polarisation experiment where the spin-locking pulse is used to transfer polarisation to less sensitive nuclei in the sample.

Although the simple vector model picture given above is valid for spin $I=1 / 2$ nuclei, the spin-locking behaviour of quadrupolar ( $\operatorname{spin} I>1 / 2)$ nuclei in solids is more challenging to understand. This is of particular significance in cross-polarisation experiments involving quadrupolar nuclei, [1, 2, 3, 4, including those where cross polarisation occurs from one quadrupolar nucleus to another, [5, 6, 7, , 8] or to a spin $I=1 / 2$ nucleus. [9, 10, 11, The quadrupolar splitting parameter, $\omega_{\mathrm{Q}}$, may be much larger than the radiofrequency field strength, resulting in rapid evolution of the magnetisation on a time scale of $1 / \omega_{\mathrm{Q}}$ before the system settles into a spin-locked state of single- and multiple-quantum coherences as well as nonequilibrium population states. Experiments performed under MAS will be more complex still, with a time-dependent quadrupolar splitting parameter and final spin-locked states which vary with the rotor phase 
$\omega_{\mathrm{R}} t .12,13,14,15$,

Previously, a simple theoretical model was presented by Ashbrook and Wimperis to describe the creation and evolution of coherences in a spin-locking experiment involving half-integer quadrupolar nuclei.[16] This model assumes that all components of the spin density operator that do not commute with the spinlocking Hamiltonian will be rapidly dephased at the start of the experiment and can be ignored. Subsequently, in a further study, the effects of secondorder quadrupolar interactions and small resonance offsets were considered.[17] In the present work, a study of the far off-resonance case will be presented. The magnetisation is left directed along the $z$-axis of the rotating frame before the spin-locking pulse is applied. 12 Since this pulse is applied well away from the Larmor frequency, there is a significant $z$ component to the effective field in the 35 rotating frame, $B_{1 \text {,eff }}$ and hence the equilibrium magnetisation has a significant component along the direction of the effective field; it is this component that is spin-locked.

The first sections of this paper will briefly recap the simple theoretical model of spin-locking based on the assumption of rapid initial dephasing. We will make extensive use of this model as its success in reproducing experimental and exact simulation data will indicate that, as with on- and near-resonance spin-locking, [16, 17] the rapid initial dephasing that takes place at the start of the spin-locking period is the key to understanding the outcome of spinlocking experiments on quadrupolar nuclei. Computer simulations employing 45 this model will then be presented, as well as exact density matrix calculations for spin $I=3 / 2$ and $I=5 / 2$. These simulations, together with experimental NMR results for the spin $I=3 / 2$ nucleus ${ }^{23} \mathrm{Na}$, will provide insight into the far off-resonance spin-locking phenomenon for half-integer quadrupolar nuclei in the solid state. 


\section{Pulse sequences for spin-locking}

Figure 1 shows the pulse sequences for the far off-resonance experiments described in this paper. Since the spin-locking pulse is applied far away from the Larmor frequency, there is a significant $z$ component to the effective field $B_{1, \mathrm{eff}}$. The magnetisation is left directed along the $z$-axis of the rotating frame before the spin-locking pulse is applied and so, unlike conventional on- (or near-) resonance spin-locking, no initial pulse is required to create a particular state first. Superficially, the pulse sequences resemble the well known two-dimensional nutation experiment. [18, 19, 20. However, it is important to note that we are not interested here in the nutation behaviour that occurs near the start of the long pulse (indeed, we specifically ignore this) but only in the time-independent states that eventually emerge. For observing the spin-locking of the central transition, a single pulse of duration $\tau$ is used, as shown in Fig. 1 1a. This pulse sequence is modified as shown in Fig. 1 10 to allow the observation of spin-locked multiple-quantum coherences created by the rapid initial dephasing. In this case

65 the second pulse is phase cycled [21, 22] to convert the desired multiple-quantum coherences into the observable single-quantum state.

\section{Theoretical model of spin-locking}

Previously, a simple theoretical model of spin locking has been introduced 16 to describe (i) the creation of a variety of coherences by rapid initial dephasing and (ii), if the experiment is performed under MAS, the evolution of these coherences on a timescale of $1 / \omega_{R}$ where $\omega_{R}$ is the MAS frequency. An outline of the model is given here. The rotating-frame Hamiltonian during an off-resonance spin-locking pulse is defined as

$$
H=H_{\mathrm{rf}}+H_{\mathrm{Q}}+H_{\Omega},
$$

where the radiofrequency pulse Hamiltonian $\left(H_{\mathrm{rf}}\right)$ and the offset Hamiltonian $75\left(H_{\Omega}\right)$ are given by,

$$
H_{\mathrm{rf}}=\omega_{1} I_{x},
$$




$$
H_{\Omega}=\Omega I_{z}
$$

The first-order quadrupolar Hamiltonian is given by

$$
H_{\mathrm{Q}}=\omega_{\mathrm{Q}}\left\{I_{z}^{2}-\frac{1}{3} I(I+1)\right\}
$$

and, assuming the axial symmetry $(\eta=0)$, the first-order quadrupolar splitting parameter is

$$
\omega_{\mathrm{Q}}=\frac{\omega_{\mathrm{Q}}^{\mathrm{PAS}}}{2}\left(3 \cos ^{2} \theta-1\right) .
$$

so where $\theta$ is the angle between the unique principal axis of the quadrupolar interaction tensor and the applied static magnetic field and

$$
\omega_{\mathrm{Q}}^{\mathrm{PAS}}=\frac{3 \pi C_{\mathrm{Q}}}{2 I(2 I-1)},
$$

with the quadrupolar coupling constant $C_{\mathrm{Q}}$ in hertz. In this work, we can ignore the second-order effects of the quadrupolar Hamiltonian as these are "offset-like" and will be dwarfed by the magnitude of the resonance offsets we 85 will be considering.

When the spin-locking pulse is applied to the initial state $\sigma(0)$, the system begins to evolve under the influence of the Hamiltonian in Eq. 1, with the timedependence given by the Liouville-von Neumann equation,

$$
\frac{\mathrm{d}}{\mathrm{d} t} \sigma(t)=-i[H, \sigma(t)]
$$

Assuming that $H$ is time-independent (and therefore ignoring any effects of 90 MAS at this stage), the solution is

$$
\sigma(t)=\exp \{-i H t\} \sigma(0) \exp \{+i H t\}
$$

A unitary transformation $V$ can be used to diagonalise the Hamiltonian,

$$
H^{D}=V H V^{-1}
$$

Equation 8 can then be written as

$$
\sigma(t)=V^{-1} \exp \left\{-i H^{D} t\right\} V \sigma(0) V^{-1} \exp \left\{+i H^{D} t\right\} V
$$


or

$$
\sigma^{\prime}(t)=\exp \left\{-i H^{D} t\right\} \sigma^{\prime}(0) \exp \left\{+i H^{D} t\right\}
$$

with

$$
\sigma^{\prime}(t)=V \sigma(t) V^{-1}
$$

The initial density operator $\sigma(0)$ is thus transformed by $V$ into the eigenbasis of the spin-locking Hamiltonian, i.e., the frame in which $H$ is diagonal. The offdiagonal elements of $\sigma^{\prime}(0)$ evolve at rates determined by $\omega_{1}$ and $\omega_{\mathrm{Q}}$ and, when considered across a powder sample where $\omega_{\mathrm{Q}}$ will vary according to crystallite orientation, they will dephase rapidly at the start of the spin-locking pulse. The diagonal elements, $\sigma^{\prime D}(0)$, are the states which commute with the spinlocking Hamiltonian; these are the states which will be spin-locked. In a static sample these states will remain unchanged for the duration $\tau$ of the spin-locking pulse. In the eigenbasis of the spin-locking Hamiltonian these are equivalent to population states. When viewed back in the normal rotating frame using

$$
\sigma(\tau)=V^{-1} \sigma^{\prime D}(0) V
$$

the elements of the final density matrix correspond to a range of coherences and population states.

Note that the removal of the off-diagonal density operator elements is a non-unitary transformation that changes the norm, $\operatorname{Tr}\left\{\sigma(t)^{2}\right\}$, of the density operator. Therefore, the simple dephasing model of spin-locking strictly only applies to experiments performed on powder samples in which nothing occurs to refocus the coherences that dephase at the start of the spin-locking period.

During MAS, the quadrupolar splitting parameter $\omega_{Q}$ becomes time dependent:

$$
\omega_{\mathrm{Q}}(t)=\frac{\omega_{\mathrm{Q}}^{\mathrm{PAS}}}{2}\left\{-\sqrt{2} \sin 2 \beta \cos \left(\omega_{\mathrm{R}} t+\xi\right)+\sin ^{2} \beta \cos 2\left(\omega_{\mathrm{R}} t+\xi\right)\right\}
$$

where $\beta$ is the angle between the unique principal axis of the quadrupolar interaction tensor and the spinning axis and $\xi$ is the initial phase about this axis. The quadrupolar splitting parameter $\omega_{\mathrm{Q}}(t)$ changes sign either two or four times 
per rotor period depending on the value of $\beta$. A measure of whether this rotordriven behaviour occurs adiabatically or suddenly in the weak-field $\left(\omega_{1} \ll \omega_{\mathrm{Q}}^{\mathrm{PAS}}\right)$ limit is given by the adiabaticity parameter introduced by Vega, 12 .

$$
\alpha=\frac{\omega_{1}^{2}}{2 \omega_{\mathrm{Q}}^{\mathrm{PAS}} \omega_{\mathrm{R}}}
$$

In the "sudden limit" $(\alpha \ll 1)$, the zero-crossing of $\omega_{\mathrm{Q}}(t)$ occurs rapidly, and the final state $\sigma(t)$ remains unchanged by MAS. In this case, the spin-locking behaviour should be the same as in a static sample. Conversely, in the "adiabatic limit" ( $\alpha \gg 1$ ), where the zero-crossing occurs slowly, the spin-locked states are time dependent. This can be incorporated into the model by using one unitary transformation, $V(0)$, to produce the initial state in the eigenbasis of the spinlocking Hamiltonian but a different transformation, $V(\tau)$, to view the density matrix in the rotating frame after a spin-locking pulse of duration $\tau, 16$

$$
\sigma(\tau)=V^{-1}(\tau)\left(V(0) \sigma(0) V^{-1}(0)\right)^{D} V(\tau) .
$$

Although the final state $\sigma(\tau)$ varies with the accumulated rotor phase $\omega_{\mathrm{R}} \tau$ as a result of this time dependence, the spins remain locked at all times. The third case, when $\alpha \sim 1$, is called the intermediate regime; here, a zero-crossing will partially convert population differences across spin-locking eigenstates into offdiagonal coherences and the spin-locked magnetisation will decay on a timescale of $\sim 1 / \omega_{\mathrm{R}}$.

\section{Spin-locking calculations}

\subsection{Spin-locking model}

This simple model of spin-locking can be utilised in numerical calculations, which we have implemented using home-written Fortran codes. The tensor operator $T_{1,0}$, which is proportional to $I_{z}$ and hence the thermal equilibrium state, forms the initial density matrix. The spin-locking pulse Hamiltonian is calculated using Equation 1. The Hamiltonian is diagonalised using the unitary transformation $V$, which is found numerically for convenience, as in Ref. [17. 
By setting the off-diagonal elements to equal zero, only the spin-locked, i.e., diagonal, elements of the density matrix are retained. The final density matrix in the rotating frame is obtained using Eq. 12 or (for MAS) 16. The final states are determined by calculating the expectation value of operator $Q$ by taking the trace of the final density matrix elements with the adjoint operator $Q^{\dagger}$,

$$
\langle Q\rangle=\operatorname{Tr}\left\{\sigma(\tau) Q^{\dagger}\right\}
$$

where $Q$ is typically a spherical tensor operator, $T_{l, p}$. We can also determine the norm of the density operator,

$$
\operatorname{Tr}\left\{\sigma(t)^{2}\right\}=\sum_{l} \sum_{p} \operatorname{Tr}\left\{\sigma(t) T_{l,-p}\right\}^{2} .
$$

The norm of a density operator is conserved under a unitary transformation. 23. 
$105.8 \mathrm{kHz}$ for ${ }^{23} \mathrm{Na}(I=3 / 2) .{ }^{23} \mathrm{Na}$ NMR studies were carried out using two compounds, sodium nitrite $\left(\mathrm{NaNO}_{2}\right)$ and sodium nitrate $\left(\mathrm{NaNO}_{3}\right)$, which were obtained commercially as powdered solids and packed in 4-mm rotors for both static and MAS experiments. In order to limit the effects of rf inhomogeneity on the experimental results, the samples were packed to a depth of around 1 $\mathrm{cm}$ only in the centre of the rotor, while the remaining volume of the rotor was packed with powdered boron phosphate as a spacer material. Each compound possesses a single crystallographically-distinct cation site, with $\omega_{\mathrm{Q}}^{\mathrm{PAS}} / 2 \pi=84$ $\mathrm{kHz}$ and $\eta=0$ for $\mathrm{NaNO}_{3}$ [24] and $\omega_{\mathrm{Q}}^{\mathrm{PAS}} / 2 \pi=275 \mathrm{kHz}$ and $\eta=0.109$ for $\mathrm{NaNO}_{2}$. 25. The central transition of sodium nitrite $\left(\mathrm{NaNO}_{2}\right)$ has a secondorder broadened MAS lineshape at $9.4 \mathrm{~T}$ with a width of $660 \mathrm{~Hz}$, but this is still small compared with the resonance offsets (up to $200 \mathrm{kHz}$ ) we consider in this work. The use of these two solid compounds, with their small and mediumsized quadrupolar coupling constants, allows us to explore a wide range of ratios of both the offset $\Omega$ and radiofrequency field strength $\omega_{1}$ to the coupling constant $\omega_{\mathrm{Q}}^{\mathrm{PAS}}$. Materials with much larger quadrupolar coupling constants would have satellite transitions that lie outside of the usable bandwidths $(1-2 \mathrm{MHz})$ of the NMR probeheads available to us and the agreement between experiment and theory, both exact and approximate, would be poor as a result of this purely instrumental limitation.

\section{Results and Discussion}

\subsection{Spin $I=3 / 2$ in a static solid}

Using the model of spin-locking described, the expectation values of the spin $I=3 / 2$ central transition single-element operator $I_{-}^{\{1 / 2,-1 / 2\}}$ and the norm, $\operatorname{Tr}\left\{\sigma(t)^{2}\right\}$, created by the rapid dephasing of the initial state $\sigma(0)=T_{1,0}$ were calculated as a function of radiofrequency field strength, $\omega_{1}$, and resonance offset, $\Omega$. Figure 2 shows the results as three-dimensional surface plots. The quadrupolar splitting parameter $\omega_{\mathrm{Q}}^{\mathrm{PAS}} / 2 \pi$ was $200 \mathrm{kHz}$ and a single orientation was chosen $\left(\beta=0^{\circ}\right.$, hence $\left.\omega_{\mathrm{Q}}=\omega_{\mathrm{Q}}^{\mathrm{PAS}}\right)$. As the radiofrequency field strength $\omega_{1}$ 
increases, Fig. 2a shows that the magnitude of the norm decreases, indicating decreasing spin-locking efficiency. The extent of the falloff with $\omega_{1}$ decreases as the resonance offset $\Omega$ increases. This is a simple consequence of the angle between the effective spin-locking field in the rotating frame and the equilibrium magnetisation increasing as $\omega_{1}$ increases but decreasing as $\Omega$ increases. At offsets equal to around $0, \omega_{\mathrm{Q}}^{\mathrm{PAS}}$ and $2 \omega_{\mathrm{Q}}^{\mathrm{PAS}}$ there are local dips in the surface plot. In Fig. 2b the central transition (CT) intensity is equal to zero when either $\omega_{1} / 2 \pi=0$ (no spin-locking pulse) or $\Omega=0$ (the on-resonance pulse does not lock the equilibrium $z$-magnetisation). The spin-locked CT intensity increases as both $\omega_{1}$ and $\Omega$ increase, with a dip in intensity at $\Omega / 2 \pi=\omega_{\mathrm{Q}}=200 \mathrm{kHz}$.

The three-dimensional surface plots in Fig. 3 show the expectation values of the spin $I=3 / 2$ spherical tensor operators for a powder sample. For simplicity, only values for tensor operators with positive coherence order $p$ are shown (although tensors with negative $p$ will also be present since the the density matrix is Hermitian). In contrast to Fig. 2, the contribution of crystallites with differing values of $\omega_{\mathrm{Q}}$ according to orientation gives rise to modified behaviour; in particular, distinctive features in the plots no longer arise only at offsets around $\omega_{\mathrm{Q}}^{\mathrm{PAS}}$ and $2 \omega_{\mathrm{Q}}^{\mathrm{PAS}}$.

The $T_{1,0}$ plot is similar to the one seen in Fig. $2 \mathrm{a}$ for the norm; the magnitude decreases as $\omega_{1}$ increases, with a more rapid falloff at lower offsets. A small dip in intensity is seen at $\Omega / 2 \pi \approx 120 \mathrm{kHz}$. $T_{2,0}$ is created with most intensity at $\Omega / 2 \pi=90 \mathrm{kHz}$ and $\omega_{1} / 2 \pi=50 \mathrm{kHz}$, while $T_{3,0}$ has its greatest intensity at lower resonance offsets and $\mathrm{rf}$ strengths, with a maximum at $\Omega / 2 \pi=5 \mathrm{kHz}$ and $\omega_{1} / 2 \pi=10 \mathrm{kHz}$.

Next considering the single-quantum spin-locked states, $T_{1,1}$ is zero when either $\omega_{1}$ and $\Omega=0$, and as these are both increased, more $T_{1,1}$ is created. $T_{2,1}$ is at a maximum when $\Omega / 2 \pi=30 \mathrm{kHz}$ and $\omega_{1} / 2 \pi=65 \mathrm{kHz}$. Both $T_{2,1}$ and $T_{3,1}$ feature intensity dips at $\Omega / 2 \pi \approx 100 \mathrm{kHz}$ at weak rf field strengths.

The magnitude and sign of the $T_{2,2}$ expectation value have a complex depen225 dence on the resonance offset and rf field strength, with a maximum magnitude at $\Omega / 2 \pi=110 \mathrm{kHz}$ and $\omega_{1} / 2 \pi=47.5 \mathrm{kHz}$. Most $T_{3,2}$ is created at $\Omega / 2 \pi=40$ 
$\mathrm{kHz}$ and $\omega_{1} / 2 \pi=97.5 \mathrm{kHz}$. The triple-quantum state $T_{3,3}$ is created with highest intensity at low offsets, with a maximum at $\Omega / 2 \pi=5 \mathrm{kHz}$ and $\omega_{1} / 2 \pi=65$ $\mathrm{kHz}$.

To confirm these model simulations, static ${ }^{23} \mathrm{Na}$ NMR spin-locking experiments have been carried out. Figure 4 shows the ${ }^{23} \mathrm{Na}$ central-transition intensity for static sodium nitrate (Fig. 4a) and sodium nitrite (Fig. 4b) as a function of increasing offset of the rf transmitter from the centre of the ${ }^{23} \mathrm{Na}$ lineshape at three different spin-locking field strengths. The experiments were recorded using the pulse sequence shown in Fig. 1, with a spin-locking pulse length equal to $\tau=300 \mu \mathrm{s}$. A recycle interval of $10 \mathrm{~s}$ was employed in the $\mathrm{NaNO}_{3}$ experiments, while a shorter interval of $1 \mathrm{~s}$ was used in the $\mathrm{NaNO}_{2}$ experiments. The experimentally-derived spin-locking intensities (datapoints shown by squares joined by a dashed line) have been empirically scaled to allow 240 a comparison with the theoretical results for the expectation value of $I_{-}^{\{1 / 2,-1 / 2\}}$ from the spin-locking model (shown by solid lines). The same scaling factor was applied to all experimental data points shown in Figs. 4 and in 5

In Fig. 4a, with the rf field strength equal to $\omega_{1} / 2 \pi=20.8 \mathrm{kHz}$, the spinlocked CT intensity for $\mathrm{NaNO}_{3}$ increases to a maximum at $\Omega / 2 \pi=50 \mathrm{kHz}$ before falling off again. With $\omega_{1} / 2 \pi=41.7 \mathrm{kHz}$, the $\mathrm{CT}$ intensity rises as the offset increases, dipping at $\Omega / 2 \pi=20 \mathrm{kHz}$ before rising to a maximum at $\Omega / 2 \pi=90 \mathrm{kHz}$. At the strongest spin-locking field, the CT intensity rises with increasing offset, dipping very slightly at $\Omega / 2 \pi=30 \mathrm{kHz}$ and reaching a maximum at $\Omega / 2 \pi=130 \mathrm{kHz}$, before falling off gradually. As $\omega_{1}$ increases, the maximum amount of spin-locked CT obtained also increases. The experimental results and the model show an excellent agreement at all three rf strengths for $\mathrm{NaNO}_{3}$. While a simulation using exact density matrix theory would also reflect the experimental results, we see here that calculations employing the simplified rapid dephasing model of spin-locking are highly successful in predicting the experimental outcome, indicating that it is the initial dephasing that dominates the experimentally observed behaviour.

Figure $4 \mathrm{~b}$ shows an increase in spin-locked CT magnetisation for $\mathrm{NaNO}_{2}$ up 
to $\Omega / 2 \pi=50 \mathrm{kHz}$ with $\omega_{1} / 2 \pi=20.8 \mathrm{kHz}$ before falling off again with a further increase in the resonance offset. At $\omega_{1} / 2 \pi=41.7 \mathrm{kHz}$, the amount of CT spinlocked builds up slowly to a maximum around $\Omega / 2 \pi=100 \mathrm{kHz}$, and remains fairly steady before decreasing at offsets greater than $140 \mathrm{kHz}$. At the strongest rf field strength, the build up of the spin-locked CT intensity as the offset increases is slower. A maximum is reached at $\Omega / 2 \pi=170 \mathrm{kHz}$. A near-perfect agreement between the experimental data and model is seen with $\omega_{1} / 2 \pi=20.8$ $\mathrm{kHz}$, although a greater divergence is seen for the higher rf strengths.

Figure 5 shows plots of the spin-locked ${ }^{23} \mathrm{Na}$ triple-quantum intensity for sodium nitrite as a function of resonance offset for three different rf field strengths. The experimental points are compared to the expectation value of the triplequantum coherence $T_{3,3}$ calculated using the simple model of spin-locking. The pulse sequence in Fig. 1 $1 \mathrm{~b}$ was used with a spin-locking pulse duration of $300 \mu \mathrm{s}$. The length of the conversion or mixing pulse was optimised for the conversion of the triple-quantum coherences back into observable central-transition coherence $\left(1.6 \mu \mathrm{s}\right.$ for $\left.\omega_{1} / 2 \pi=166.7 \mathrm{kHz}\right)$.

With the spin-locking rf field strength set to $83.3 \mathrm{kHz}$, the amount of triplequantum coherence rises rapidly as the offset increases up to $\Omega / 2 \pi=7 \mathrm{kHz}$. The intensity then declines as the offset is raised further. Halving the rf strength to $41.7 \mathrm{kHz}$, the maximum amount of triple-quantum coherence is created at a lower offset of $\Omega / 2 \pi=2 \mathrm{kHz}$. When the field strength is lowered further to $20.8 \mathrm{kHz}$, the occurrence of triple-quantum coherence is again shifted to a still lower offset, with a maximum at $\Omega / 2 \pi=1 \mathrm{kHz}$. It is clear that, except at high rf field strengths, little TQ intensity arises in a static solid as a result of the far off-resonance spin-locking experiment.

For the two strongest rf strengths, the calculations closely match the experimental results, although both slightly underestimate the amount of spinlocked magnetisation present at higher offset. The calculation carried out with $\omega_{1} / 2 \pi=20.8 \mathrm{kHz}$ also significantly underestimates the amount of spin-locked triple-quantum magnetisation created at low offsets. However, the general trend is still reflected accurately. 


\subsection{Spin $I=3 / 2$ under $M A S$}

5 , there is little $T_{3,3}$ created by the initial dephasing at this magnitude of offset but that more is created by adiabatic transfer during the rotation of the sample.

Next, considering the plots in Figure 6b where a larger offset of $100 \mathrm{kHz}$ has been used, we see that there is a smaller amplitude for the modulation of the

the population states $T_{1,0}, T_{2,0}$ and $T_{3,0}$ are present in greatest intensity in order of decreasing rank. This is to be expected in view of the initial state being the $p=0$ population state $T_{1,0}$ in this type of far off-resonance experiment. The decrease in intensity of these leads to the rotor-driven interconversion with the 
most significant. A large decrease is observed in the intensities of $T_{3,1}, T_{3,2}$ and $T_{3,3}$ compared with the simulations for $\Omega / 2 \pi=50 \mathrm{kHz}$ in Fig. 6a.

Density matrix calculations confirm the validity of the results found using the spin-locking model. In Fig. 7, the spin $I=3 / 2$ expectation values have been calculated in a powder over two rotor periods using (a) the dephasing model and (b) an exact density matrix approach. The oscillations seen before in Fig. 6 are strongly dependent on the crystallite orientation as defined in Eq. 3 , while here the summation of the orientations over the full range of $\beta$ and $\xi$ values offers a more realistic representation of a typical experiment on a powder. After 330 a period of initial rapid dephasing (the noise-like oscillations on the left of the plots), the states settle into a periodic interconversion of spin-locked states in Fig. 7b, principally between populations and single-quantum coherences as suggested by Fig. 6. The calculation using the simple model (Fig. 7 a) shows excellent agreement in predicting the evolution of the spin-locked states.

${ }^{23} \mathrm{Na}$ MAS NMR spin-locking experiments have been performed on $\mathrm{NaNO}_{2}$, confirming the complex rotor-driven dynamics predicted by theory. Figure 8 shows experimental ${ }^{23} \mathrm{Na}$ spin-locked central-transition (Figs. 8a and 8b) and triple-quantum (Figs. 8c and 8d) intensities as a function of the spin-locking pulse length, where the maximum pulse length was equal to five full rotor periods $\left(500 \mu \mathrm{s}\right.$ at a MAS frequency of $\left.\omega_{\mathrm{R}} / 2 \pi=10 \mathrm{kHz}\right)$. In Fig. 8 the spin-locking field strength used was $83.3 \mathrm{kHz}$, yielding an adiabaticity parameter of $\alpha=1.26$. In this adiabatic limit, the rotor-driven modulation is clearly apparent. As the rotor turns, the $\mathrm{CT}$ intensity increases, reaching a maximum after a half turn. This is followed by a decrease during the second half of the rotor period as the various crystallites experience zero-crossings. At the end of each rotor period, the crystallites have experienced either two or four zero-crossings, and the original signal intensity is re-established. In Fig. 8b, the spin-locking field strength was reduced to $20.8 \mathrm{kHz}$, yielding $\alpha=0.08$. In these experiments, the modulation of the eigenstates falls into the sudden limit and the effect of sample 
seen in Figs. 8c and 8d, where the spin-locked triple-quantum coherence is monitored as a function of spin-locking pulse duration in the adiabatic limit (Fig. 8c) and the sudden limit (Fig. 8d). In the adiabatic limit, an obvious modulation of the intensity is observed, with minima at integer rotor periods. 355 This corresponds to the simulations where population states are most abundant at the start of each rotor period, and sample rotation induces coherence-transfer to higher order states. When performed at a lower spin-locking field strength (Fig. 8d), there is no obvious strong modulation of the amplitude with rotation.

\subsection{Spin $I=5 / 2$ under $M A S$}

360 matrix calculation is presented in Fig. 9. Here the evolution of the spin-locked states has been calculated for the duration of two complete turns of the rotor. The same simulation parameters are used as described for the corresponding spin $I=3 / 2$ simulation (Fig. 7), giving an adiabaticity parameter of $\alpha=1.4$ for the density matrix calculation. For the population states and single-quantum coherences, a good qualitative agreement can be seen between the model and the exact density matrix simulations. However, for the higher order coherences, the magnitude of the spin-locked states decays on the timescale of around $1 / \omega_{R}$ in the density matrix simulations, such that there is little magnetisation spinlocked in these states during the second rotor period. This behaviour is also observed in conventional or near-resonance spin-locking[17] for spin $I=5 / 2$ and appears to be a consequence of the greater number of spin transitions blurring the distinction between the "adiabatic" and "sudden" spin-locking regimes originally defined by Vega[12] for spin $I=3 / 2$. Purely adiabatic behaviour is 35 known[17] to be difficult to observe for spin $I=5 / 2$, whatever the value of the adiabaticity parameter, and there always appears to be some degree of dephasing on a timescale of $1 / \omega_{R}$. Our simplified model of spin-locking, which assumes that a rapid initial dephasing takes place and that spin-locking under MAS is then entirely in either the sudden or adiabatic regimes, is not able to reproduce this level of detail.

A comparison of the spin $I=5 / 2$ results of the model with an exact density 


\section{Conclusions}

Using the model of quadrupolar spin-locking introduced by Ashbrook and Wimperis [16] which predicts the spin-locked state after an initial period of rapid dephasing, we have investigated the far off-resonance case of spin locking

for spin $I=3 / 2$ and $I=5 / 2$ nuclei. Since the offset is large, there is a significant $z$-component to the effective field $B_{1 \text {,eff }}$ and so the magnetisation is left directed along the rotating-frame $z$-axis before the pulse is applied. We have made extensive use of this simplified model as its success in reproducing experimental and exact simulation data indicates that, as with on- and nearresonance spin-locking, [16, 17] the rapid initial dephasing that takes place at the start of the off-resonance spin-locking period is the key phenomenon in such experiments performed on quadrupolar nuclei.

Three-dimensional surface plots showing the creation of a range of different coherences as a function of offset and spin-locking field strength have been presented. In the plots for single-orientation nuclear spin, features occur in the plots at integer multiples of $\omega_{\mathrm{Q}}^{\mathrm{PAS}}$ (see Fig. 2), while more complicated behaviour is observed in the powder simulations (Fig. 3). Spin $I=3 / 2$ calculations using the dephasing model were found to be successful in predicting the results of single-quantum and triple-quantum experiments carried out on $\mathrm{NaNO}_{2}$ and $\mathrm{NaNO}_{3}$ (Figs. 4 and 5 ).

Under MAS, a time dependence is introduced to the quadrupolar splitting parameter, and the spin-locking behaviour in the weak-field limit $\left(\omega_{1} \ll \omega_{\mathrm{Q}}\right)$ falls into different regimes according to the value of the adiabaticity parameter $\alpha$, as defined by Vega. [12] In the adiabatic limit, $\alpha \gg 1$, the spin-locked states also acquire a time dependence. The rotor-driven interconversion of eigenstates predicted by the rapid dephasing model calculations are reflected by the bruteforce density matrix simulations in Figs. 7 and 9 .

In the sudden limit, $\alpha \ll 1$, the spin-locked states under MAS are expected to be similar to those found in static solids. This was found in Figs. 8b and 8d, where experiments on $\mathrm{NaNO}_{2}$ show little variation in the spin-locked single- or 
triple-quantum central transition amplitudes with MAS.

As in our previous investigations of the spin-locking of quadrupolar nuclei, the rapid dephasing model has been shown to explain virtually all of the key features in far-off resonance spin-locking experiments found both experimentally and in exact density matrix calculations. Relaxation effects are clearly not included in either the exact or approximate theoretical models used here but are unlikely to be important on experimentally accessible timescales unless significant dynamics are present in the solid. The close agreement between theory and experiments in this work is noteworthy. Far off-resonance spin-locking of quadrupolar nuclei requires an initial state (the thermal equilibrium bulk magnetisation) that is created solely by spin-lattice and spin-spin relaxation during the long relaxation interval. In contrast, if a central-transition selective pulse is applied to create the initial state, as in conventional spin-locking, that initial state will be much less well defined in a powder as a consequence of the impossibility of the condition $\omega_{1} \ll \omega_{\mathrm{Q}}$ holding for all crystallites. Therefore, although possibly only of esoteric interest, we believe that far off-resonance spin-locking could be relevant to experiments such as cross polarisation from one quadrupolar nucleus to another, [5, 6, 7, , 8, or to a spin $I=1 / 2$ nucleus, 9, 10, 11] as we would expect better agreement with any prior theoretical investigation. Off-resonance cross polarisation has been investigated before for spin $I=1 / 2$ nuclei[26] and is not generally considered to be a useful technique in that application but the particular features of cross polarisation involving quadrupolar nuclei 3 may make it worthwhile re-examining.

\section{Acknowledgements}

We are grateful to EPSRC for a studentship (SO) and to Professor Sharon Ashbrook (St Andrews, UK) for useful discussions about spin-locking of quadrupolar nuclei. 


\section{Funding}

This research did not receive any specific grant from funding agencies in the 440 public, commercial, or not-for-profit sectors.

\section{References}

[1] R. K. Harris, G. J. Nesbitt, Cross polarization for quadrupolar nuclei: proton to sodium-23, Journal of Magnetic Resonance 78 (1988) 245-256.

[2] S. Ding, C. A. McDowell, Theoretical calculations of the CPMAS spectral lineshapes of half-integer quadrupole systems, Journal of Magnetic Resonance A 114 (1995) 80-87.

[3] S. Ashbrook, S. Wimperis, Single- and multiple-quantum cross-polarization in NMR of quadrupolar nuclei in static samples, Molecular Physics 98 (2000) $1-26$.

[4] M. Edén, Quadrupolar coupling selective cross-polarization in solid state NMR, Physical Chemistry Chemical Physics 8 (2006) 1994-1999.

[5] L. van Wüllen, L. Züchner, W. Müller-Warmuth, H. Eckert, ${ }^{11}{ }^{1}-{ }^{27} \mathrm{Al}$ and ${ }^{27} \mathrm{Al}-{ }^{11} \mathrm{~B}$ double-resonance experiments on a glassy sodium alumino-borate, Solid State Nuclear Magnetic Resonance 6 (1996) 203-212.

[6] J. C. C. Chan, M. Bertmer, H. Eckert, Double-quantum cross-polarization between half-integer quadrupolar spin systems: ${ }^{11} \mathrm{~B}-{ }^{23} \mathrm{Na}$ and ${ }^{11} \mathrm{~B}-{ }^{27} \mathrm{Al}$, Chemical Physics Letters 292 (1998) 154-160.

[7] J. C. C. Chan, M. Bertmer, H. Eckert, Site connectivities in amorphous materials studied by double-resonance nmr of quadrupolar nuclei: Highresolution ${ }^{11} \mathrm{~B}_{-}{ }^{27} \mathrm{Al}$ spectroscopy of alumino-borate glasses, Journal of the American Chemical Society 121 (1999) 5238-5248.

[8] J. C. C. Chan, High-resolution heteronuclear correlation between quadrupolar nuclei, Journal of Magnetic Resonance 140 (1999) 487-490. 
[9] C. A. Fyfe, H. Grondey, K. T. Mueller, K. C. Wong-Moon, T. Markus, Coherence transfer involving quadrupolar nuclei in solids: ${ }^{27} \mathrm{Al}-{ }^{31} \mathrm{P}$ crosspolarization NMR in the molecular sieve VPI-5, Journal of the American Chemical Society 114 (1992) 5876-5878.

[10] S. M. De Paul, M. Ernst, J. S. Shore, J. F. Stebbins, A. Pines, Crosspolarization from quadrupolar nuclei to silicon using low radio-frequency amplitudes during magic-angle spinning, Journal of Physical Chemistry B 101 (1997) 3240-3249.

[11] R. Siegel, J. Rocha, L. Mafra, Combining STMAS and CRAMPS NMR spectroscopy: High-resolution HETCOR NMR spectra of quadrupolar and ${ }^{1} \mathrm{H}$ nuclei in solids, Chemical Physics Letters 470 (2009) 337-341.

[12] A. J. Vega, MAS NMR spin locking of half-integer quadrupolar nuclei, Journal of Magnetic Resonance 96 (1992) 50-68.

[13] W. Sun, J. T. Stephen, L. D. Potter, Y. Wu, Rotation-induced resonance and second-order quadrupolar effects on spin locking of half-integer quadrupolar nuclei, Journal of Magnetic Resonance 116 (1995) 181-188.

[14] G. Jeschke, Spin locking of $I=3 / 2$ nuclei in static and spinning samples: A description by abstract spins and Floquet formalism, Journal of Chemical Physics 108 (1998) 907-917.

[15] Y. Zhang, F. Deng, J. Qui, C. Ye, Spin-locking mechanism of spin $I=$ $3 / 2$ quadrupolar nuclei undergo magic angle spinning, Solid State Nuclear Magnetic Resonance 15 (2000) 209-216.

[16] S. Ashbrook, S. Wimperis, Spin-locking of half-integer quadrupolar nuclei in nuclear magnetic resonance of solids: Creation and evolution of coherences, Journal of Chemical Physics 120 (2004) 2719-2731.

[17] S. Ashbrook, S. Wimperis, Spin-locking of half-integer quadrupolar nuclei in nuclear magnetic resonance of solids: Second-order quadrupolar and resonance offset effects, Journal of Chemical Physics 131 (2009) 194509. 
[18] A. P. M. Kentgens, J. J. M. Lemmens, F. M. M. Geurts, W. S. Veeman, Two-dimensional solid-state nutation nmr of half-integer quadrupolar nuclei, Journal of Magnetic Resonance 71 (1987) 62-74.

[19] A. Samoson, E. Lippmaa, 2d nmr nutation spectroscopy in solids, Journal of Magnetic Resonance 79 (1988) 255-268.

[20] A. P. M. Kentgens, Off-resonance nutation nuclear magnetic resonance spectroscopy of half-integer quadrupolar nuclei, Progress in Nuclear Magnetic Resonance Spectroscopy 32 (1998) 141-164.

[21] G. Bodenhausen, H. Kogler, R. R. Ernst, Selection of coherence transfer pathways in NMR pulse experiments, Journal of Magnetic Resonance 58 (1984) 370-388.

[22] P. J. Hore, J. A. Jones, S. Wimperis, NMR: The Toolkit (2nd edition), Oxford University Press, 2015.

[23] O. W. Sørensen, Polarization transfer experiments in high-resolution NMR spectroscopy, Progress in Nuclear Magnetic Resonance Spectroscopy 21 (1989) 503-569.

[24] A. P. M. Kentgens, A practical guide to solid-state NMR of half-integer quadrupolar nuclei with some applications to disordered systems, Geoderma 80 (1997) 271-306.

[25] E. Kundla, A. Samoson, E. Lippmaa, High-resolution NMR of quadrupolar nuclei in rotating solids, Chemical Physics Letters 83 (1981) 229-232.

[26] A. Bax, B. L. Hawkins, G. E. Maciel, Off-resonance cross-polarization: A technique to reduce rf power requirements for magnetization transfer experiments in solids, Journal of Magnetic Resonance 59 (1984) 530-535. 
(a)

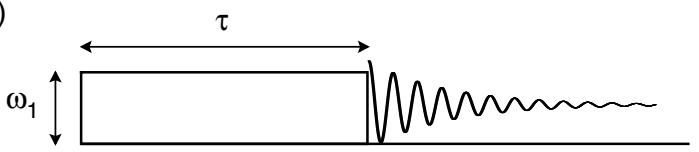

(b)

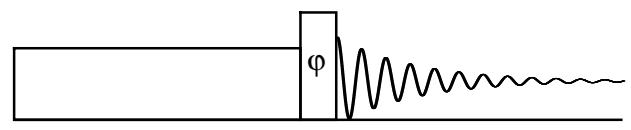

Figure 1: Pulse sequences for observing spin-locked (a) single-quantum and (b) multiplequantum coherences under far off-resonance conditions.
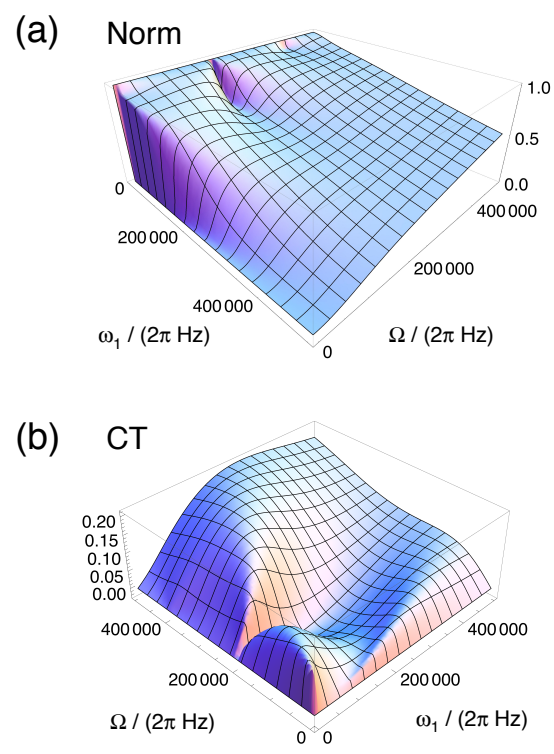

Figure 2: Three-dimensional surface plots of the spin $I=3 / 2$ expectation values for (a) the density operator norm and (b) the central transition (CT) created by rapid dephasing of the initial state $T_{1,0}$ under spin-locking, as a function of $\omega_{1}$ and $\Omega$. Results are plotted for a single orientation $(\theta=0)$ with $\omega_{\mathrm{Q}}^{\mathrm{PAS}} / 2 \pi=200 \mathrm{kHz}$. 

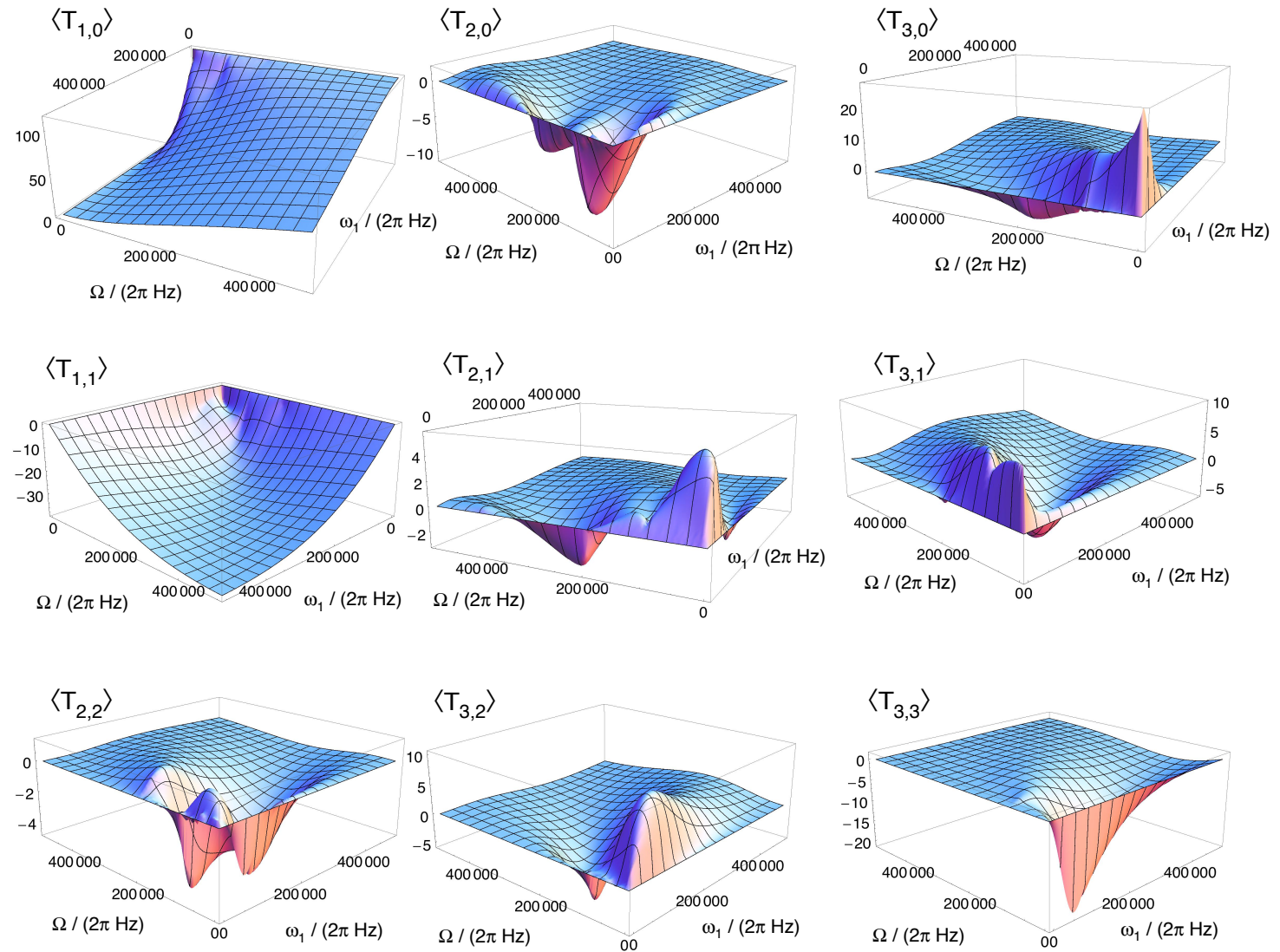

Figure 3: Three-dimensional surface plots of the spin $I=3 / 2$ expectation values of spherical tensor operators, $T_{l, p}$ created by rapid dephasing of initial state $T_{1,0}$ under a spin-locking Hamiltonian, as a function of $\omega_{1}$ and $\Omega$. Results are plotted for a powder sample with $\omega_{\mathrm{Q}}^{\mathrm{PAS}} / 2 \pi=200 \mathrm{kHz}$. 

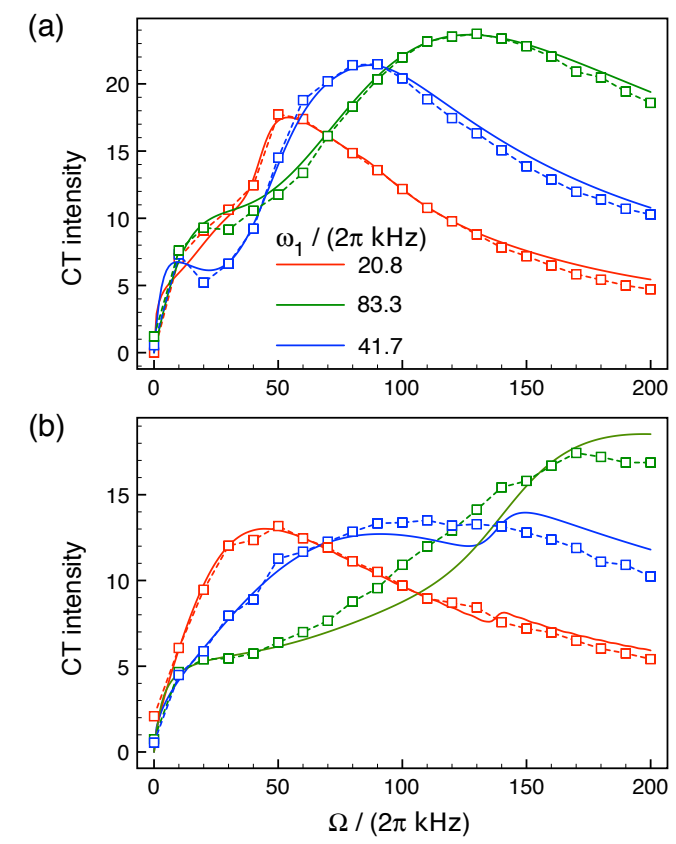

Figure 4: Spin-locked ${ }^{23} \mathrm{Na} \mathrm{NMR}$ central-transition intensity in (a) $\mathrm{NaNO}_{3}$ and (b) $\mathrm{NaNO}_{2}$ as a function of resonance offset, recorded using the pulse sequence in Fig. 1 1 . The spin-locking pulse duration was $300 \mu \mathrm{s}$ in each case. Experimental points are shown by squares joined by a dashed line, while the spin-locking intensities calculated using the spin-locking model are shown by a solid line. The intensities of all experimental points were multiplied by the same empirical constant to allow comparison with the calculated spin-locking intensities. 

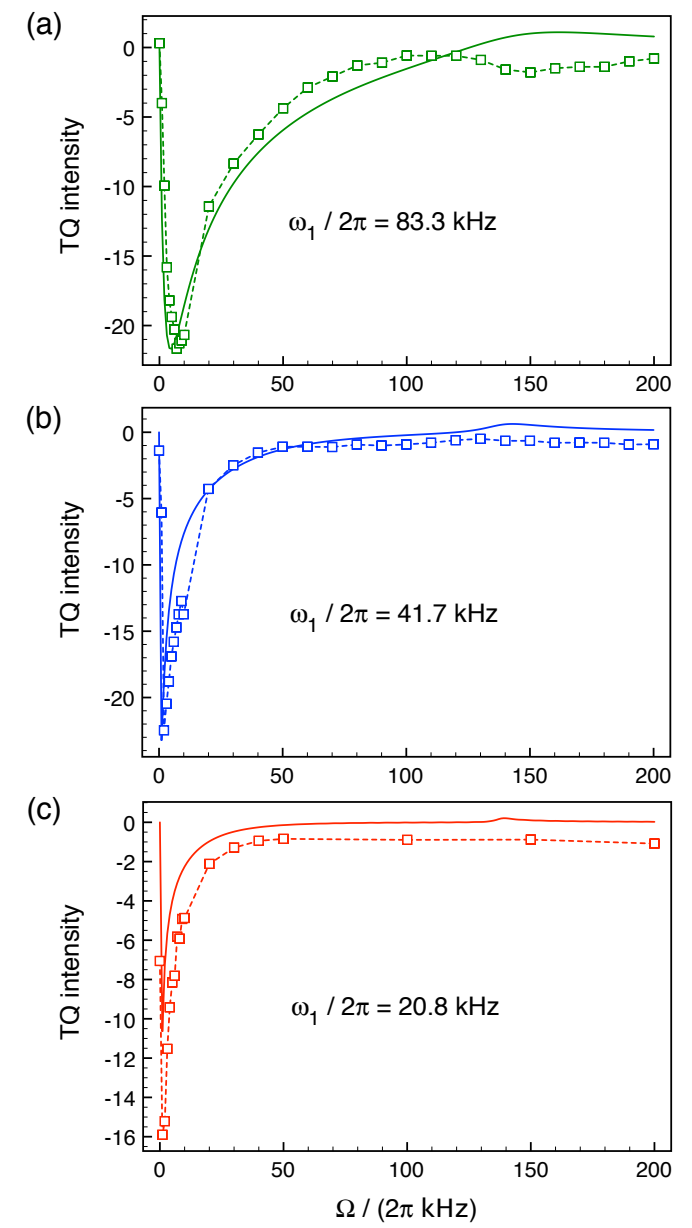

Figure 5: Spin-locked ${ }^{23} \mathrm{Na} \mathrm{NMR}$ triple-quantum intensity in $\mathrm{NaNO}_{2}$ as a function of resonance offset, recorded using the pulse sequence in Fig. 1 1 b for spin-locking rf field strengths of (a) $83.3 \mathrm{kHz}$, (b) $41.7 \mathrm{kHz}$ and (c) $20.8 \mathrm{kHz}$. The spin-locking pulse duration was $300 \mu \mathrm{s}$ in each case. Experimental points are shown by squares joined by a dashed line, while the spin-locking intensities calculated using the spin-locking model are shown by a solid line. 
(a)
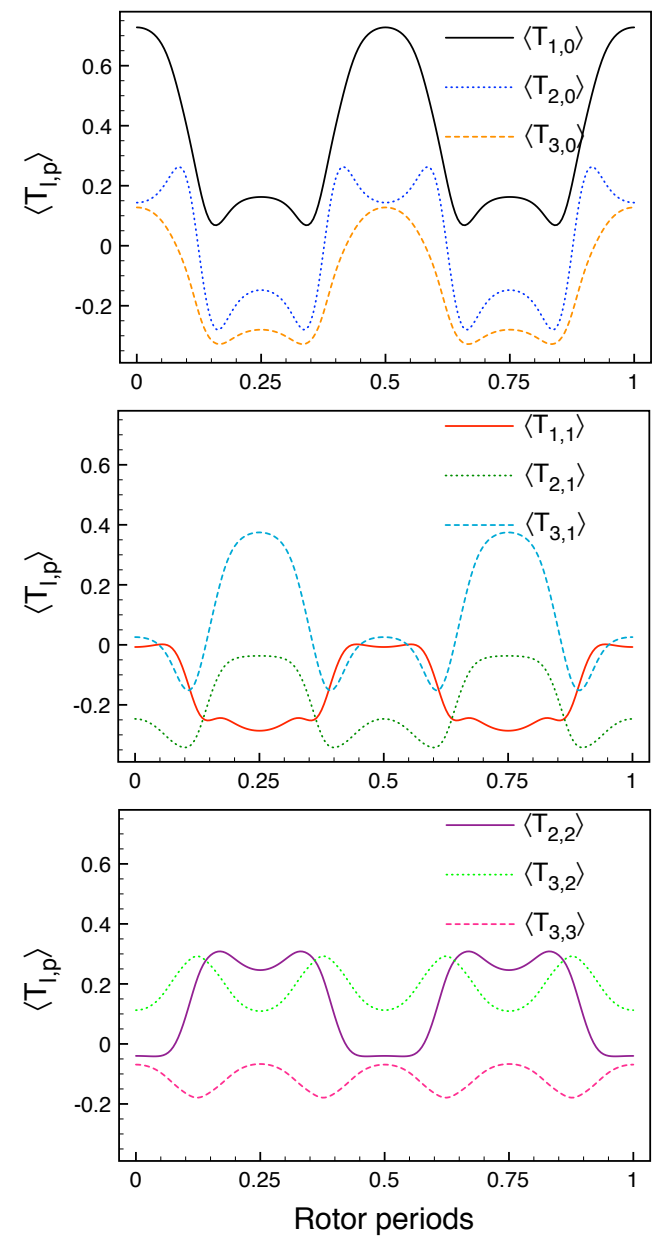

(b)
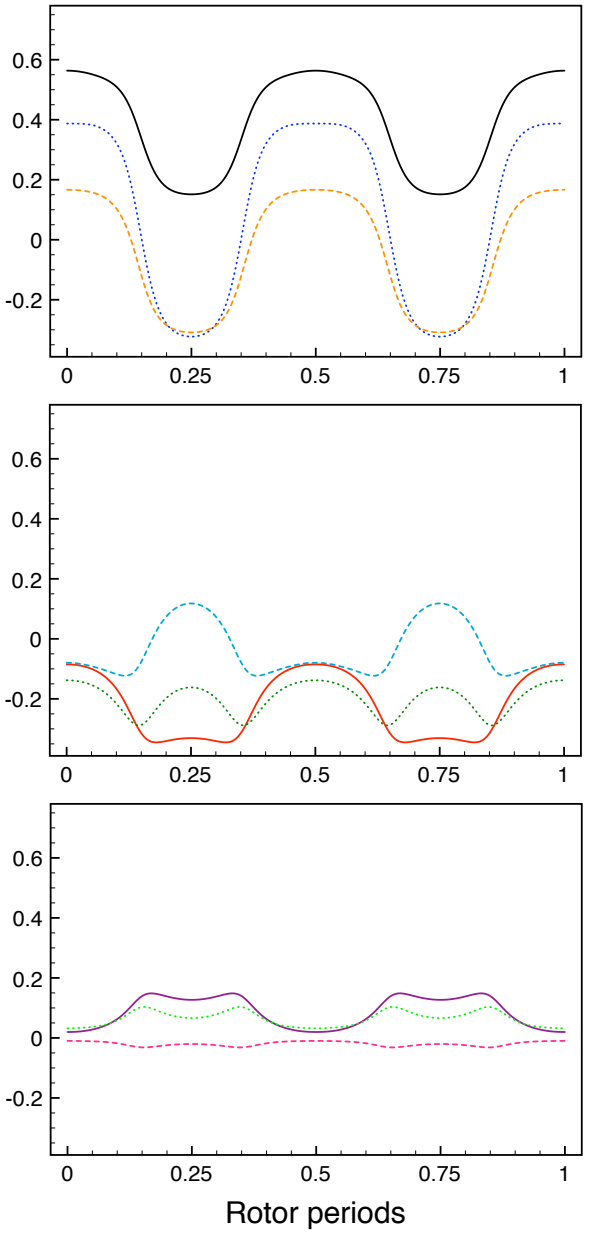

Figure 6: Expectation values of $I=3 / 2$ spherical tensor operators, $T_{l, p}$, created by rapid dephasing of an initial state $T_{1,0}$ under spin-locking and MAS. The resonance offset was (a) $\Omega / 2 \pi=50 \mathrm{kHz}$ and (b) $\Omega / 2 \pi=100 \mathrm{kHz}$. Results are plotted as a function of rotor period for a single crystallite orientation $\left(\beta=90^{\circ}\right.$ and $\left.\xi=180^{\circ}\right)$ with $\omega_{1} / 2 \pi=75 \mathrm{kHz}$ and $\omega_{\mathrm{Q}}^{\mathrm{PAS}} / 2 \pi=200 \mathrm{kHz}$. 
(a)
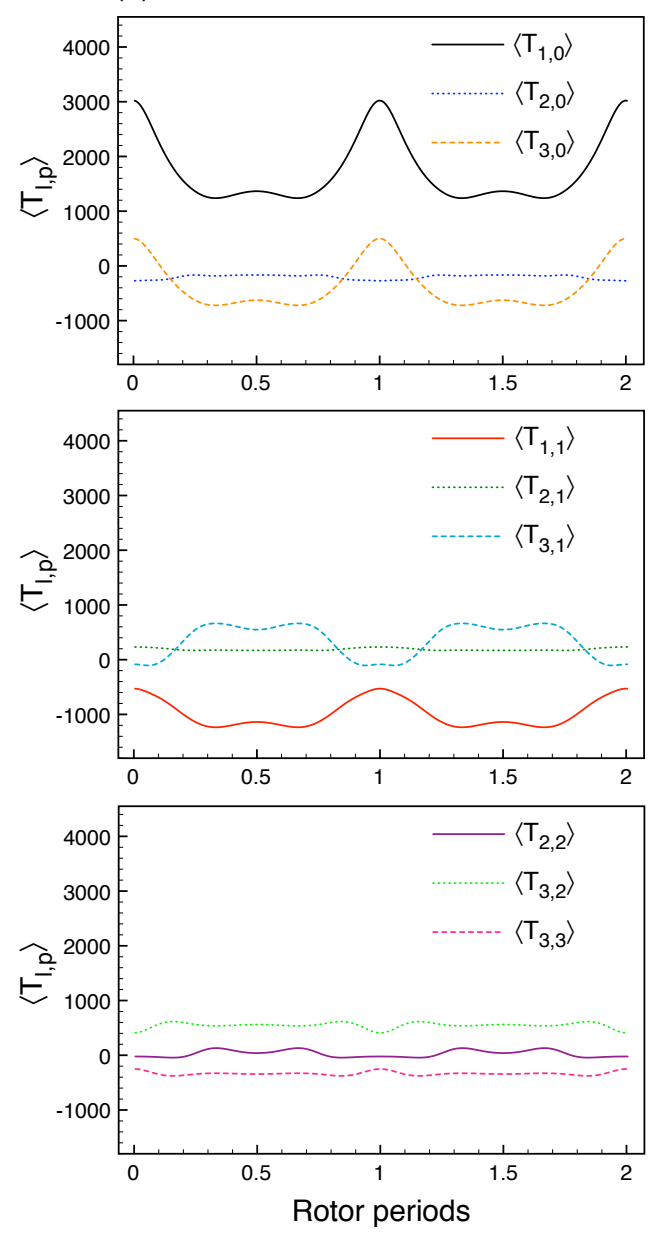

(b)
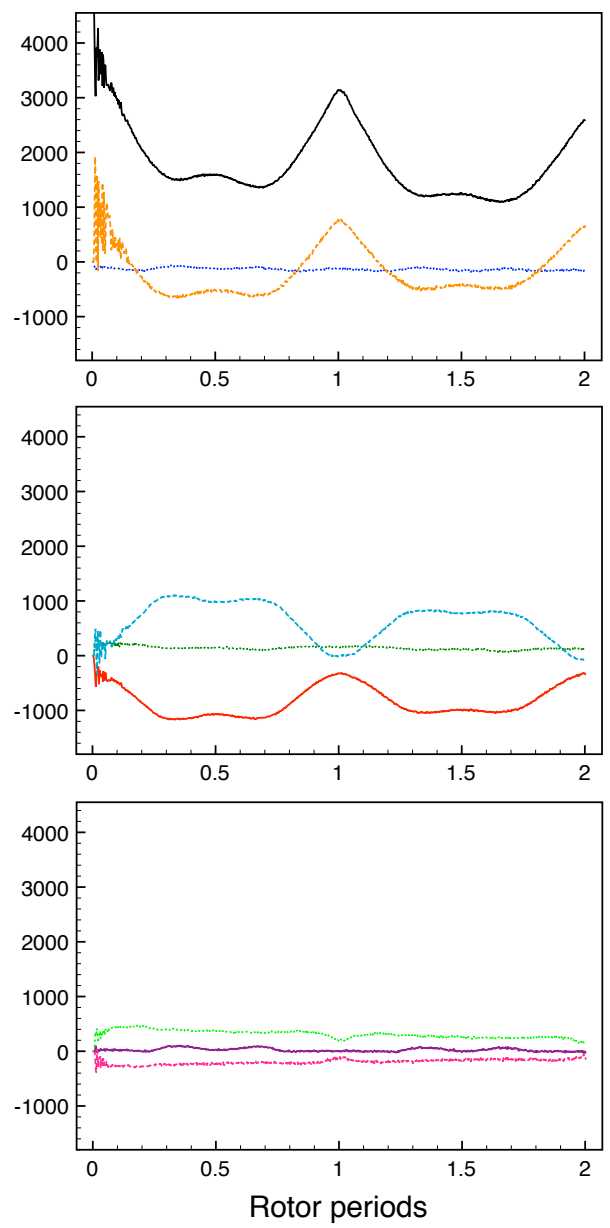

Figure 7: Evolution of expectation values of $I=3 / 2$ spherical tensor operators, $T_{l, p}$, under MAS. Results are plotted for a powder with $\omega_{\mathrm{Q}}^{\mathrm{PAS}} / 2 \pi=200 \mathrm{kHz}, \omega_{1} / 2 \pi=75 \mathrm{kHz}$ and $\Omega / 2 \pi=50 \mathrm{kHz}$. Results are shown for (a) the spin-locking model and (b) an exact density matrix calculation. The MAS rate in (b) was $\omega_{\mathrm{R}} / 2 \pi=10 \mathrm{kHz}$, yielding an adiabaticity parameter of $\alpha=1.4$ for the density matrix calculations. 
(a)

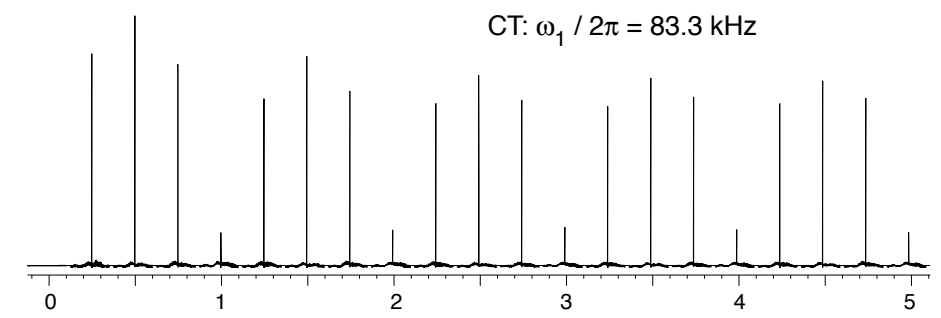

(b)

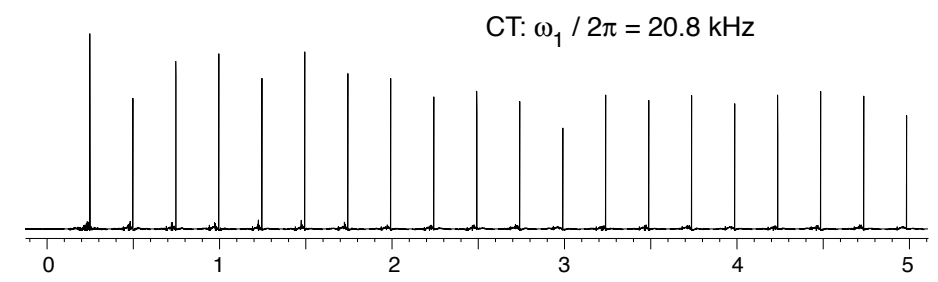

(c)

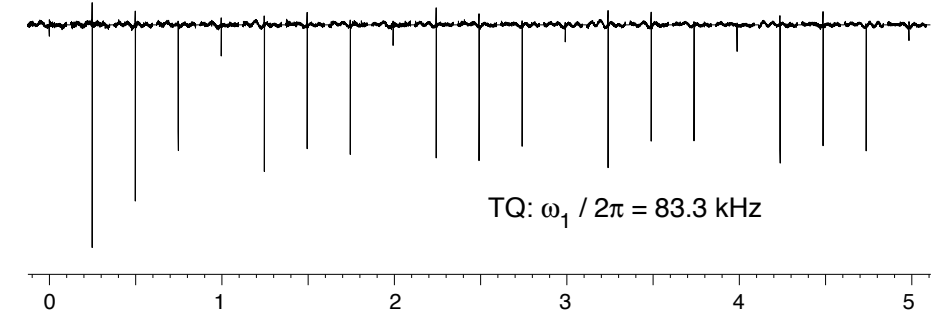

(d)
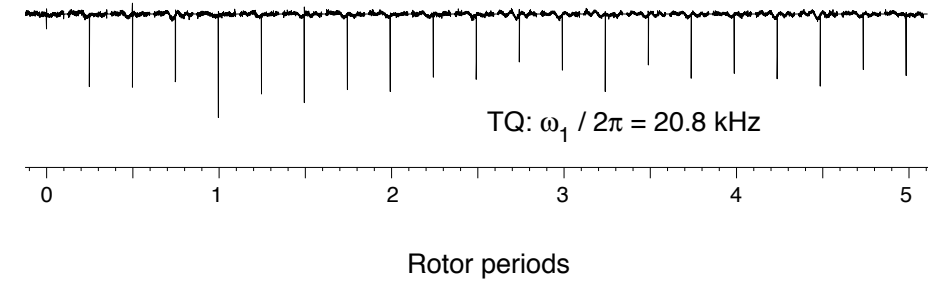

Figure 8: ${ }^{23} \mathrm{Na}$ MAS NMR experiments, showing the signal intensity of the $(\mathrm{a}, \mathrm{b})$ central transition and (c, d) triple-quantum coherence during the spin-locking of $\mathrm{NaNO}_{2}$ at a resonance offset of $50 \mathrm{kHz}$ with varying spin-locking durations. The MAS rate was $\omega_{\mathrm{R}} / 2 \pi=10$ $\mathrm{kHz}$, yielding adiabaticity parameters of $(\mathrm{a}, \mathrm{c}) \alpha=1.26$ and $(\mathrm{b}, \mathrm{d}) \alpha=0.08$. 
(a)
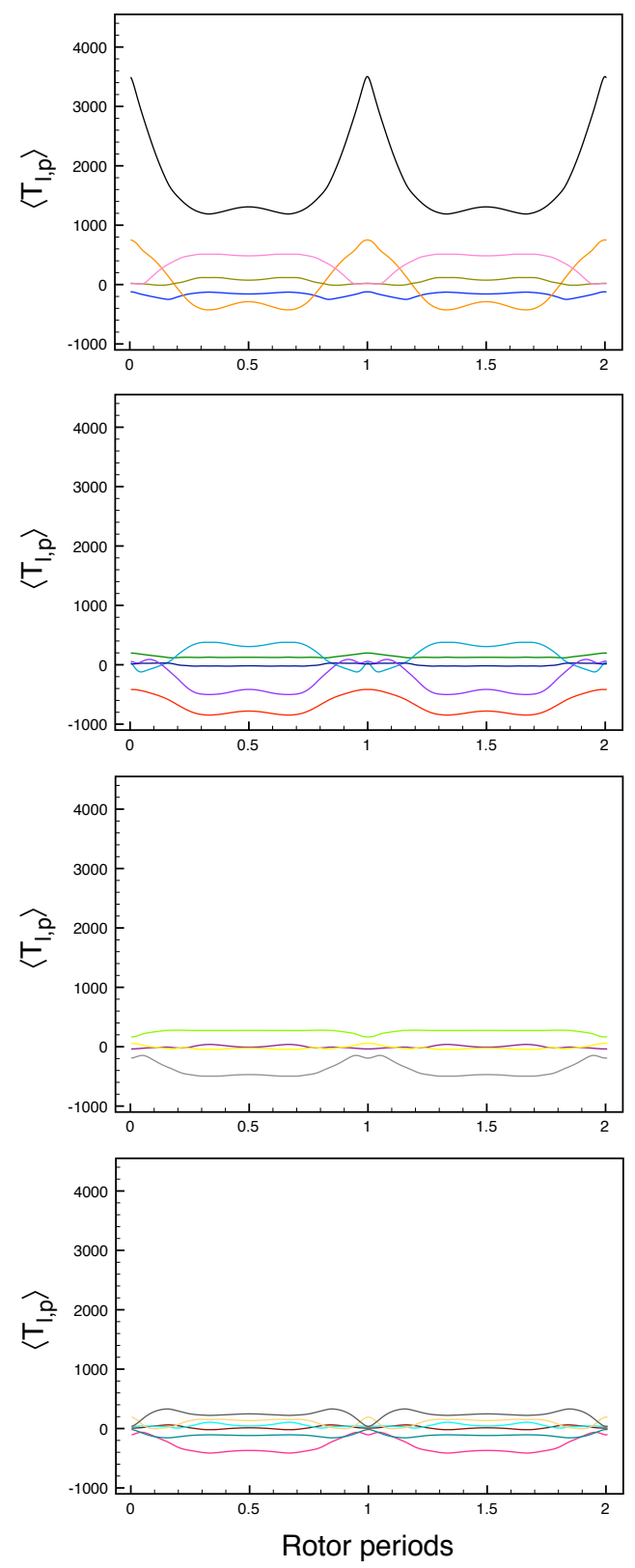

(b)
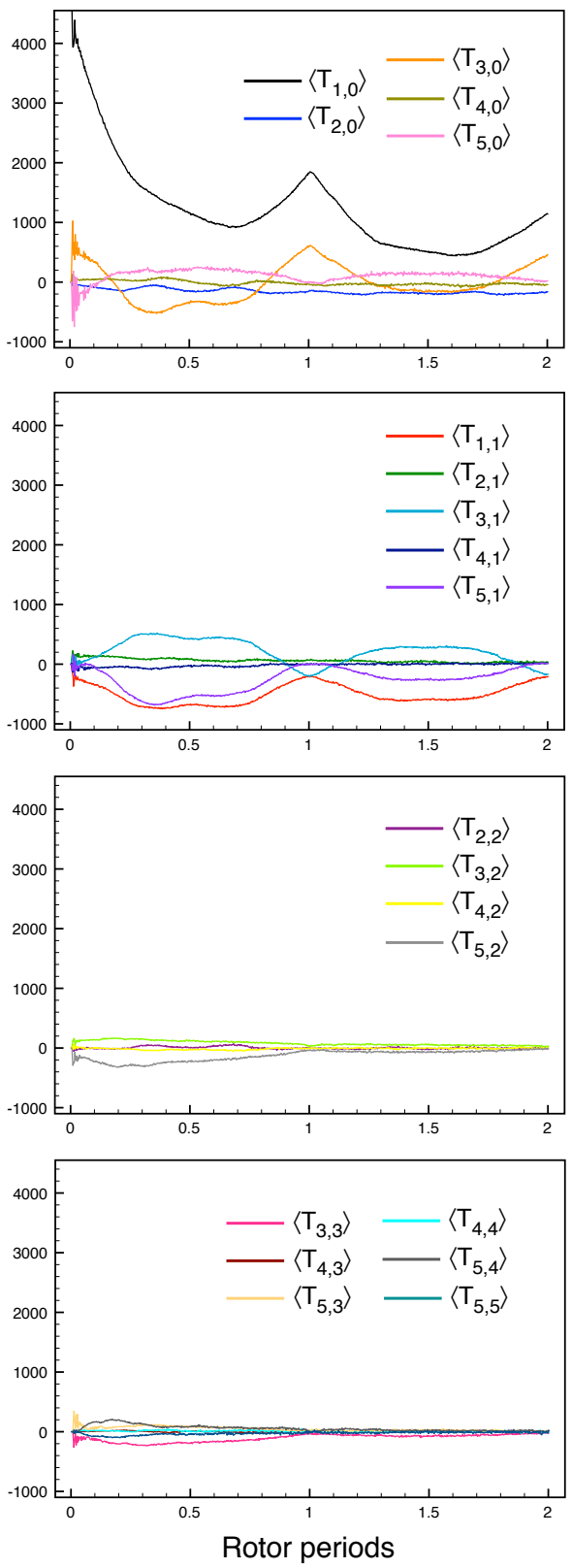

Figure 9: Evolution of expectation values of $I=5 / 2$ spherical tensor operators, $T_{l, p}$, under MAS. Results are plotted for a powder with $\omega_{\mathrm{Q}}^{\mathrm{PAS}} / 2 \pi=200 \mathrm{kHz}, \omega_{1} / 2 \pi=75 \mathrm{kHz}$ and $\Omega / 2 \pi=50 \mathrm{kHz}$. Results are shown for (a) the spin-locking model and (b) an exact density matrix calculation. The MAS rate in (b) was $\omega_{\mathrm{R}} / 2 \pi=10 \mathrm{kHz}$, yielding an adiabaticity parameter of $\alpha=1.4$ for the density matrix cegculations. 